Axel Boese*, and Michael Friebe

\title{
Is a thin diameter ureteroscope feasible for image guided intravascular procedures?
}

\begin{abstract}
Vascular endoscopic imaging is known for a long time but has never made its way into clinical routine. Reasons for that are the complexity, lack of low-cost portable systems, and the lack of suitable endoscopes providing high image quality with small dimensions. In addition, an interruption of the blood flow caused by the device and the opacity of blood are difficult to manage. In the past we have already developed ideas to overcome these difficulties and now we present a feasibility test of a thin diameter ureteroscope for observation of vascular procedures. The imaging system was tested in a phantom where side branches were explored, a stent was placed and a simulated aneurysm coiled.
\end{abstract}

Keywords: angioscopy, optical vascular imaging, endoscopy, stenting, coiling, vascular access

https://doi.org/10.1515/cdbme-2020-3151

\section{Introduction}

Endoscopic imaging is used in several medical disciplines for diagnostic and treatment monitoring. It is intuitive, since the presented information is very close to the natural human cognition. But vascular endoscopy (angioscopy) is rarely used in intra vascular procedures even though there are clear diagnostic benefits [1]. Angioscopy allows for example the assessment of plaques, vessel wall dissections or implants. But the opacity of the blood itself prevents a free view to the structures of interest. There have been a few approaches to realize intra vascular endoscopy. One option is the use of a specific catheter with an optical image fiber inside a balloon. By inflating the balloon and pressing it onto the vessel wall the blood is shifted away allowing a clear view to the vessel wall. This method is used for example to observe laser based ablation [2]. Another method to achieve clear view inside blood vessels is based on the blockage of the blood flow. The blood in front of an inflated balloon catheter is removed by

\footnotetext{
*Corresponding author: Axel Boese: Otto-von-Guericke University Magdeburg, Medical Faculty, Leipziger Str. 44, Magdeburg, Germany, e-mail: axel.boese@ovgu.de Michael Friebe: Otto-von-Guericke University Magdeburg, Medical Faculty, Magdeburg, Germany
}

flushing with saline solution or contrast agent [1] to clear the field of view.

Optical coherence tomography (OCT) is a different optical imaging method used in the vascular system but suffers from the same problem of the blood opacity. For OCT the blood is removed shortly by a constant high-flow flush of saline solution or contrast agent during image acquisition. Thus, no blockage of the artery is needed [3]. Recent research showed the feasibility of a combination of OCT and angioscopy in one session. One flush is used for acquisition of structural and surface information of the vessels [4], [5].

Another challenge for angioscopy is the selection of the appropriate endoscopy system. Conventional systems are expensive, bulky and are not easy to integrate into the workflow of vascular treatment procedures. A small and lightweight system would be required, that can acquire high quality videos and can be connected to and displayed on the screens of the angiography suite [6]. Due to the small diameter and the tortuosity of most vessels, small dimensions and high flexibility of the endoscopes are required. This is unfortunately contrary to the achievable image quality [7]. Mostly fibre endoscopes are applied for angioscopy. Image quality and flexibility of these endoscopes are highly related to the number of fibres inside the bundle. Miniaturisation of electronics will possibly allow the use of chip on the tip cameras in the future leading to better steerability of the endoscope tip and increased image quality [8].

Angioscopy is not only a tool for diagnostic. It can also be used for observation of treatment procedures. Compared to X-Ray based fluoroscopic imaging it provides a realistic visualisation of the procedure and can reduce radiation exposure. Therefore, not only an endoscope is needed, but in addition, a second channel for tool access is necessary. This working channel can be integrated in the endoscope or can be created by a second catheter placed in parallel to it.

In this paper we show the feasibility test of a thin standard ureteroscope with integrated working channel for observation of the placement of vascular implants. The integrated bending function of the endoscope is used to support access into bifurcations under direct optical image guidance. 


\section{Materials}

\subsection{Creation of a test sample}

To test the approach of optical image guided intravascular procedures we first created an artificial test sample. Therefore, an inner core of the sample was produced out of tubes $(\varnothing 4 \mathrm{~mm})$ and hot glue. One bifurcation and a simulated aneurysm $(\varnothing$ $6 \mathrm{~mm}$, sidewall) were modeled. The core structure was preshaped using a $2 \mathrm{~mm}$ aluminum wire inside the tube. For modelling the phantom, a two-component silicon rubber (GLOREX RTV, Switzerland) was used. At all three ends connection tubes for flushing and sealing were integrated. The creation of the test sample can be seen in Figure 1.
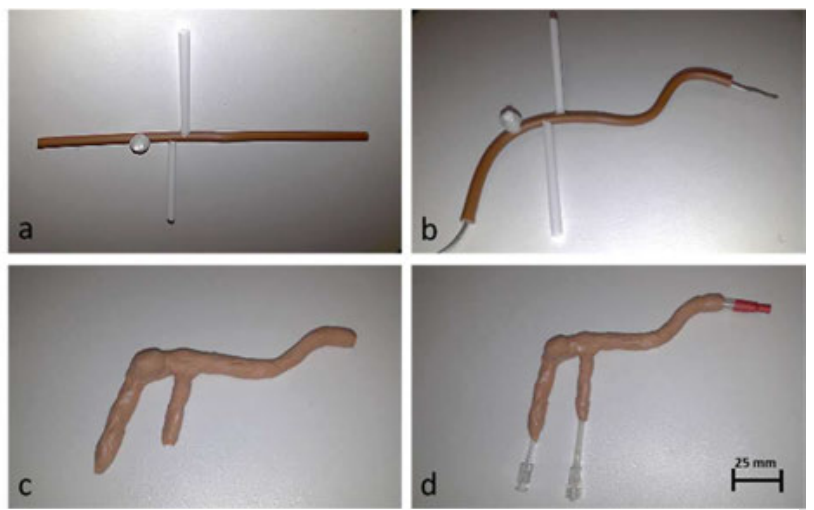

Figure 1: Creation of the test sample a) inner core with bifurcation and aneurysm, b) pre-shaping of inner structure, c) modelling using silicon rubber, d) integration of connection tubes for flushing and access

\subsection{Test setup}

A standard endoscopy system (EVIS EXERA III, Olympus, Germany) combined with a thin ureteroscope (URF-P7, Olympus, Germany) was used for image acquisition. This fiber endoscope offers optical, light and working channel and a bending mechanism while having an outer diameter of only $2,65 \mathrm{~mm}$. The diameter of the working channel of $1,2 \mathrm{~mm}$ allows passage of standard microcatheters or other vascular devices. It is used in combination with an introducer sheet that acts like a guide catheter. The specifications of the ureteroscope are shown in Table 1. Figure 2 shows a close up view of the bendable endoscope tip.

For the test setup the introducer sheet is placed inside the proximal entry of the phantom. The ureteroscope is fed into the sheet. The phantom is filled with saline solution. Air bubbles are removed by flushing with a syringe on the distal ends of the phantom. The test setup is shown in Figure 3.

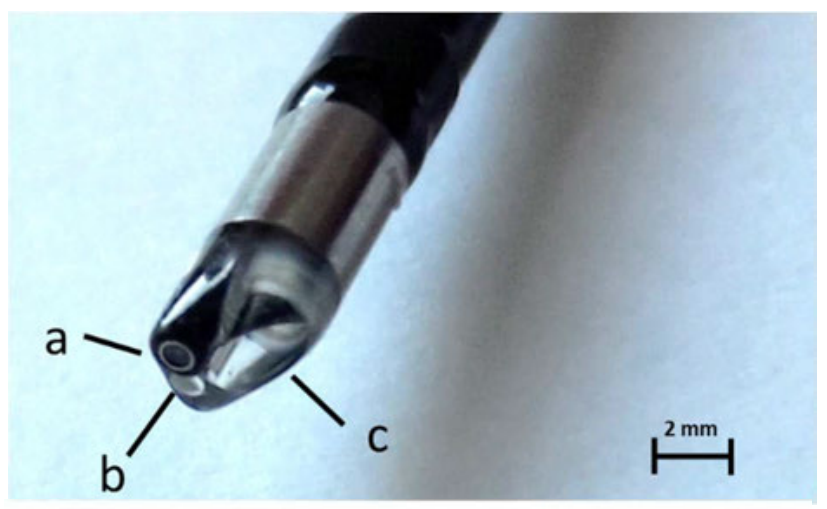

Figure 2: Tip of the endoscope: a) image channel, b) light channel, c) working channel

Table 1: Specifications of Olympus ureteroscope URF-P7

\begin{tabular}{lll}
\hline Optical system & $\begin{array}{l}\text { Field of view } \\
\text { Direction of view } \\
\text { Depth of field }\end{array}$ & $\begin{array}{l}90^{\circ} \\
\text { Forward viewing } \\
2-50 \mathrm{~mm}\end{array}$ \\
\hline Insertion tube & $\begin{array}{l}\text { Distal end outer } \\
\text { diameter } \\
\text { Insertion tube outer } \\
\text { diameter } \\
\text { Working length }\end{array}$ & $\begin{array}{l}4.9 \mathrm{Fr} \text { EvolutionTip } \\
7.95 \mathrm{Fr}(2.65 \mathrm{~mm})\end{array}$ \\
\hline Instrument channel & Inner channel diameter & $3.6 \mathrm{Fr}(1.2 \mathrm{~mm})$ \\
\hline Bending section & Angulation range & $\mathrm{Up} 275^{\circ}, \mathrm{Down} 275^{\circ}$ \\
\hline Total length & & $1050 \mathrm{~mm}$ \\
\hline
\end{tabular}

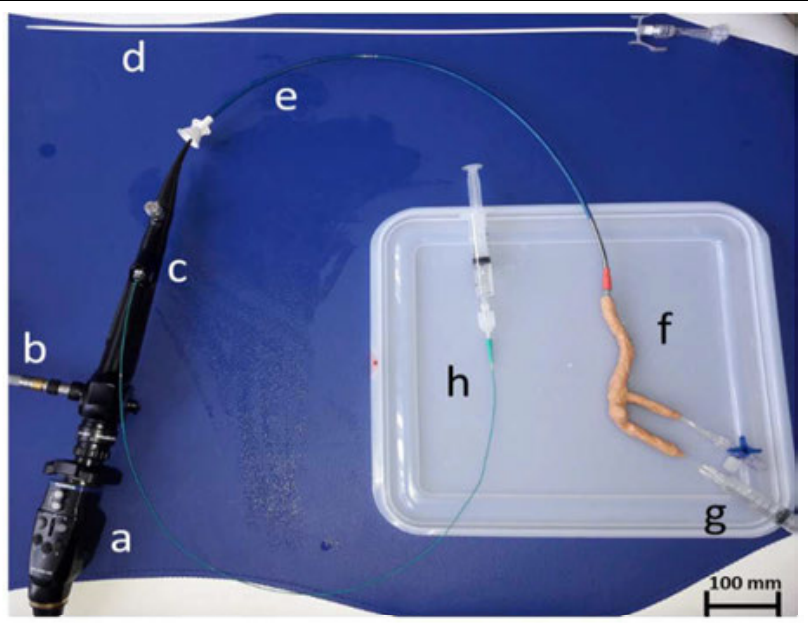

Figure 3: The test setup: a) endoscopic camera, b) light cable, c) endoscope, d) dilatator, e) introducer sheet, f) test model, g) syringe for flushing, h) catheter

For the tests standard vascular devices made for coronary or neurovascular procedures are used. An overview of these devices is given in Table 2 .

Table 2: Vascular test devices

\begin{tabular}{llll}
\hline Device & Provider & Type & \\
\hline Guide Wire & EV3 neurovascular & SilverSpeed 14 & \\
\hline Micro catheter & EV3 neurovascular & Rebar 18 2,4F & \\
\hline $\begin{array}{l}\text { Balloon expandable } \\
\text { stenting system }\end{array}$ & BBraun & Coroflex Blue 2,75x13 & \\
\hline $\begin{array}{l}\text { Detachable coil } \\
\text { system }\end{array}$ & EV3 neurovascular & $\begin{array}{l}\text { Morpheus 3D } \\
\text { 8mm CSR }\end{array}$ & \\
\hline
\end{tabular}




\subsection{Testing}

Four different tests were conducted under optical image guidance and supported by the bending function of the endoscope. The ureteroscope was placed in a distance of $10 \mathrm{~mm}$ before a branch in the side wall. In the first test maneuver this side branch was accessed by a pre-shaped guide wire and a balloon expandable catheter system. To support that, the ureteroscope tip was slightly bended into the direction of the branching vessel.

A balloon dilatation and stent placement including removal of catheter and guidewire were performed as a second maneuver.
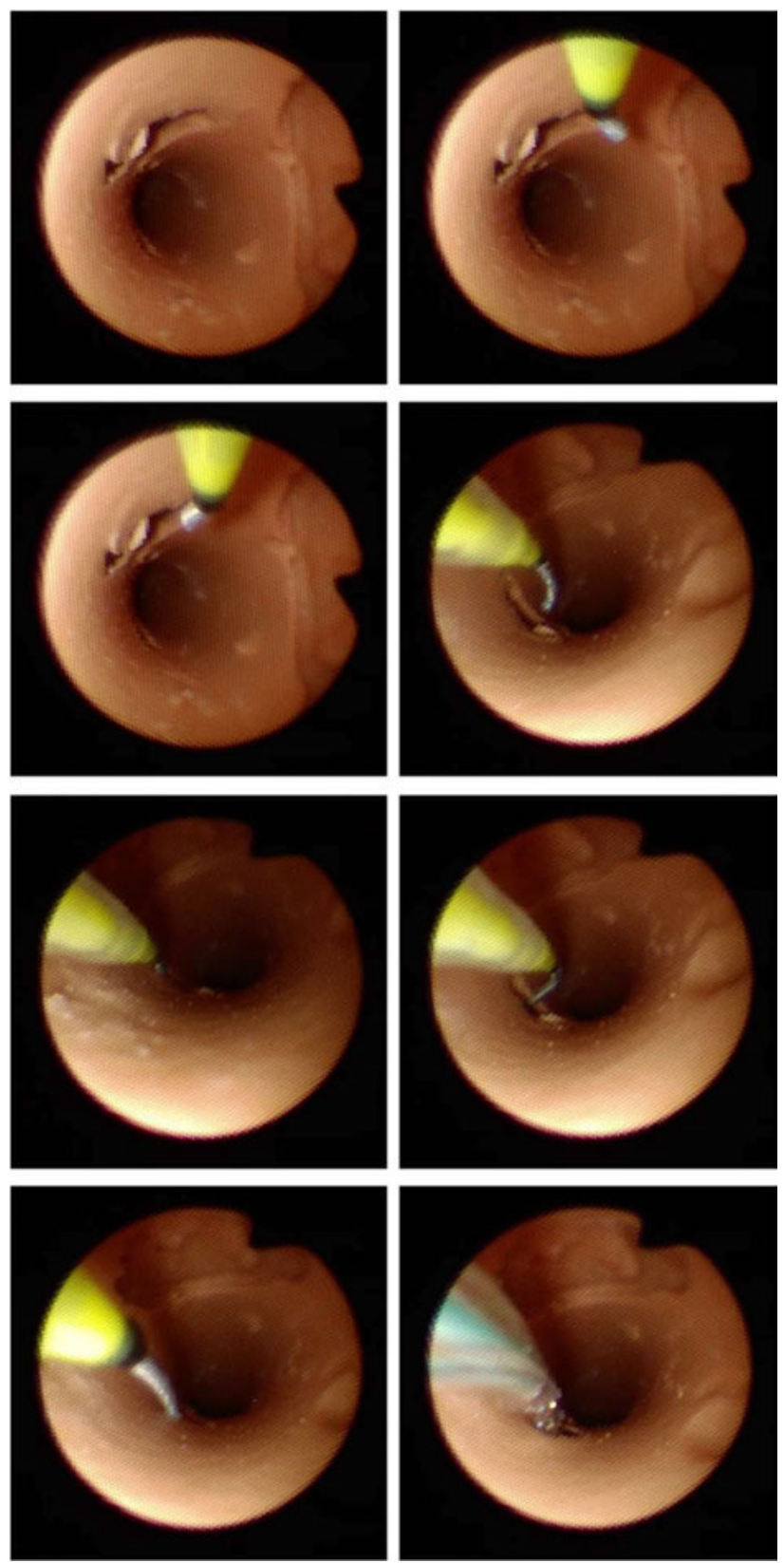

Figure 4: Endoscopic view; workflow of guidewire and catheter placement in a side branch
In a next step, the endoscope was fed deeper into the phantom to localize the aneurysm. Access of the aneurysm neck with a micro catheter was recorded as the third maneuver. Finally, the aneurysm was filled with a coil as a last maneuver.

All procedures were recorded using a screen capture device on the endoscopic system. The time from beginning of the maneuver till successful placement was recorded for all procedures.

\section{Results and discussion}

Figure 4 shows some snap shots of the placement of the guide wire and catheter in a side branch. After endoscope placement catheter and guide wire were fed into the working channel until both appear in front of the endoscope tip. The guide wire with pre-shaped tip is rotated into the direction of the branch. Supported by the bending mechanism of the endoscope, the guide wire tip is pushed into the branch and then fed into it. The catheter easily follows the guide wire into the vessel. Tendencies of the guide wire to slip out can be recognized by the optical imaging and counteract by the bending mechanism of the endoscope.
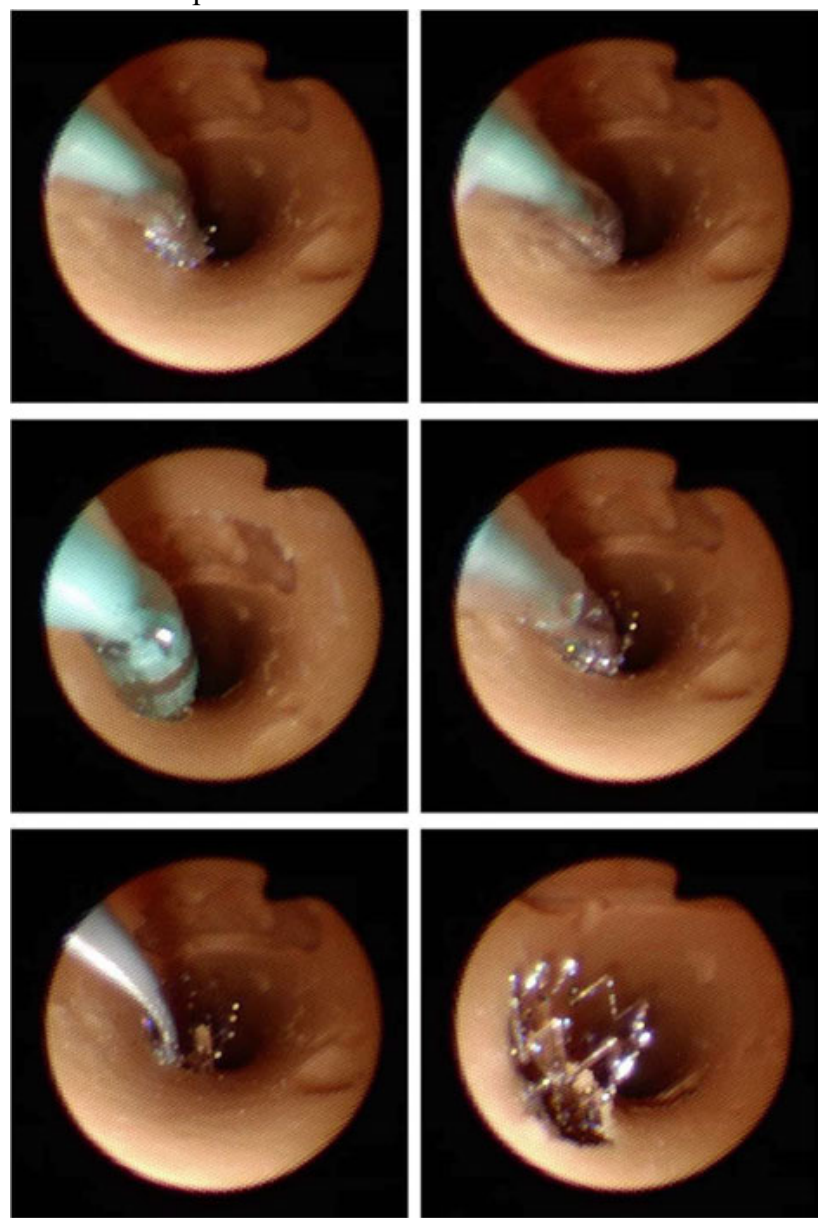

Figure 5: Dilatation and stenting of the side branch under optical observation 
The maneuver of balloon dilatation and stenting is shown in Figure 5. The balloon is filled with saline solution and increases its diameter up to $3 \mathrm{~mm}$. At the same time the stent is expanded. After reduction of the balloon the catheter and guide wire were removed successively.

The access to the aneurysm and coiling procedure is shown in Figure 6. Coiling can be seen in distant and close up view. The bending mechanisms was used to support the coiling as well.
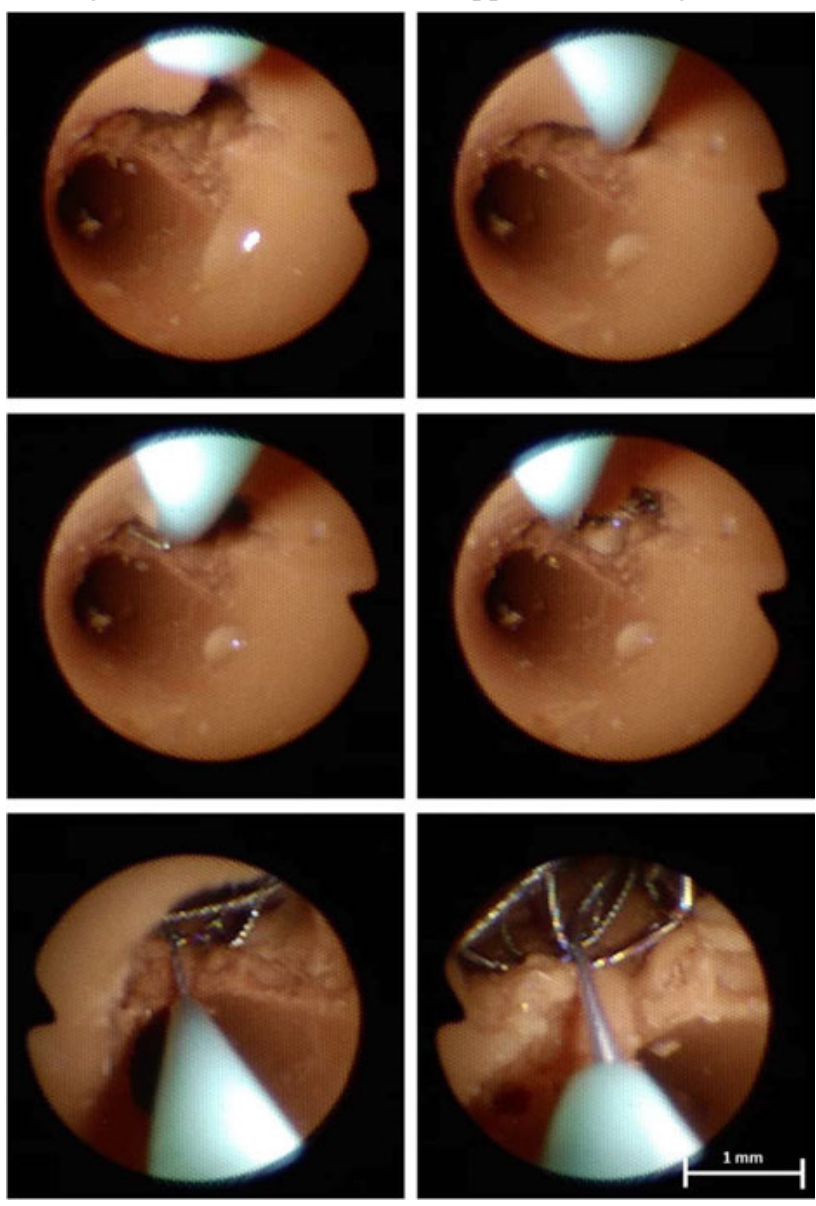

Figure 6: The test setup: a) camera, b) filter box, c) laser, d) test sample

The recorded times for the described test maneuvers are fast compared to X-Ray guidance and are summarized in Table 3.

Table 3: Times for the shown procedures

\begin{tabular}{ll}
\hline Procedure & Time \\
\hline Side branch access & $28 \mathrm{~s}$ \\
\hline Stenting & $75 \mathrm{~s}$ \\
\hline Coiling access & $22 \mathrm{~s}$ \\
\hline Coiling & $124 \mathrm{~s}$ \\
\hline
\end{tabular}

\section{Conclusion}

Feasibility and potential of optical image guided intravascular procedures were shown in this paper. The endoscope is small enough to be utilized in several vascular procedures. Techniques of blockage and flushing to remove blood are accepted for various applications. The image quality of the system used is high but images will be blurrier in reality due to mixture of the flushing liquid with blood particles. Even though angioscopy adds more complexity to vascular procedures, it has a potential of providing better visual feedback to perform interventions. Additionally, due to the intuitive presentation of the scene it has the potential to reduce procedure and radiation time.

\section{Author Statement}

Research funding: The author states no funding involved. Conflict of interest: Endoscope provided by Olympus.

\section{References}

[1] F. Ishibashi et al, „Update on Coronary Angioscopy: Review of a 20-Year Experience and Potential Application for Detection of Vulnerable Plaque“, Journal of Interventional Cardiology, Bd. 19, Nr. 1, S. 17-25, 2006,

[2] S. R. Dukkipati u. a., „Visual Balloon-Guided Point-by-Point AblationClinical Perspective“, Circulation: Arrhythmia and Electrophysiology, Bd. 3, Nr. 3, S. 266-273, Juni 2010, doi: 10.1161/CIRCEP.109.933283.

[3] T. Kume und S. Uemura, „Current clinical applications of coronary optical coherence tomography“, Cardiovasc Interv Ther, Bd. 33, Nr. 1, S. 1-10, Jan. 2018,

[4] A. Boese, A. Sivankutty, und M. Friebe, „Optical endovascular imaging by combination of endoscopy and OCT“, in 53rd Annual Conference of the German Society for Biomedical Engineering, Frankfurt, Germany, 2019.

[5] A. Boese, A. K. Sivankutty, und M. Friebe, „Optical endovascular imaging combining endoscopy, NBI and OCT, a feasibility study“, Current Directions in Biomed. Engineering, doi:10.1515/cdbme2019-0145.

[6] A. Boese und M. Friebe, ,SMARTSCOPE - portable, easy to use and cheap smartphone endoscopic system", in Abstracts 51st annual conference of the German Society for Biomedical Engineering, Dresden, Germany, 2017,

[7] A. Boese, A. K. Sivankutty, und M. Friebe, „Evaluation and image quality comparison of ultra-thin fibre endoscopes for vascular endoscopy“, Current Directions in Biomedical Engineering, Bd. 3, Nr. 2, S. 231-233, 2017, doi: 10.1515/cdbme-2017-0048.

[8] A. Boese, C. Arens, und M. Friebe, „Concept of a flexible endoscope with swiveling camera tip", in 54th Annual Conference of the German Society for Biomedical Engineering (BMT 2020), Leipzig, Germany, Sep. 2020 